Original Paper

\title{
Effect of Solvents on the Surface Modification of Hydrophilic Macro-Porous Particles with an Ion-Exchange Monomer Having Both Anion and Cation Exchange Groups
}

\author{
Kanami SHIBATA ${ }^{1}$, Tetsuya TANIGAWA ${ }^{1}$, Haruka ITO ${ }^{1}$, Takuya KUBO ${ }^{2}$, Ken HOSOYA*1 \\ ${ }^{1}$ Graduate School of Life and Environmental Science, Kyoto Prefectural University, \\ 1-5 Shimogamo-Hangicho, Sakyo-ku, Kyoto 606-8522, Japan \\ ${ }^{2}$ Graduate School of Engineering, Kyoto University, Katsura, Nshigyo-ku, Kyoto 615-8510, Japan
}

\begin{abstract}
An ion-exchange monomer including an anion exchange group and a cation exchange group, 2-methacryloyloxyethylphosphoryl choline (MPC) was introduced on the surface of uniformly sized, hydrophilic macro-porous polymer particle. We carefully studied several solvents utilized for the surface modification to elucidate solubility of the monomer as well as its polymer into each solvent. Based on the change in existing form of poly-MPC modified on the surface of stationary phases, ion-exchange properties of poly-MPC were controllable to result in interesting selectivity changes in HPLC.
\end{abstract}

Keywords: Ion-exchange group; Uniformly sized particle; Surface modification; MPC

\section{Introduction}

In late years, ecosystem effects of chemical contaminants having really low concentration in environmental water have been seriously concerned. These compounds have quite hydrophilic properties, namely Pharmaceuticals and Personal Care Products (PPCPs). Majority of PPCPs includes hydroxyl groups, carboxylic acids, and/or amine functional groups, therefore, the analyses for PPCPs necessitate quite difficult and time-consuming pre-treatment processes [1-3].

Due to the really hydrophilic properties of PPCPs, commercially available chromatographic columns as well as solid phases for the extraction for various purposes lack of utilities [4-7]. Considering the pre-treatment processes, PPCPs should be concentrated on the solid phase from aqueous media, and retained through the following washing processes using methanol or acetonitrile. For this purpose, ion-exchange group is thought to be suitable that is simply because in hydrophilic organic solvent, such as acetonitrile, hydrophilic interaction chromatography mode effectively works to result in removal of hydrophobic contaminants,

${ }^{*}$ Corresponding author: Ken HOSOYA

Tel: +81-75-703-5444; Fax: +81-75-703-5444

E-mail: hosoya@kpu.ac.jp while the target compound retained.

We have reported surface modified, uniformly sized polymer-based stationary phases for selective concentration of hydrophobic organic pollutants, such as dioxins or bisphenol A. The selectivity was achieved by the surface modification with a monomer having sulfonic acid to exclude humic acids in environmental water due to the ionic repulsion. As just described, ion-exchange groups are effective for not only retention of ionic compounds but also exclusion of contaminants [8].

An ion-exchange monomer, 2-methacryloyloxyethylphosphoryl choline (MPC) includes an anionic and a cationic functional group in it (Fig. 1).

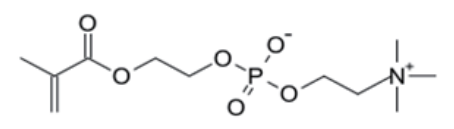

Fig. 1. Monomer; 2-methacryloyloxyethyl-phosphoryl choline (MPC).

Received: 1 April 2016

Accepted: 1 June 2016

J-STAGE Advance Published: 8 June 2016

DOI: $10.15583 /$ jpchrom.2016.004 
MPC does not have any counter ions, because interactions between at least two monomers deny the charge each other. This phenomenon clearly suggests that introduction form of MPC will be controlled by the change of polymerization conditions of MPC. In this study, we have selected glycerol dimethacrylate (GDMA) based, macroporous particles as a base material. MPC was introduced by a radical polymerization in various solvents to control the introduction form of poly-MPC on the base material. Further chromatographic evaluation will elucidate utility of MPC to retain a variety of highly hydrophilic compounds.

\section{Experimental}

\subsection{Reagents and solvents}

Reagents and solvents were utilized without further purification unless otherwise specified, while ultrapure water utilized in this study was purified with a Milli-Q Gradient A10 (Merck Millipore, Tokyo, Japan). Styrene utilized for the preparation of seed polymer particles was purchased from Wako Pure Chemicals (Osaka, Japan). GDMA was kindly donated by Shin-Nakamura Chemical Co., Ltd (Wakayama, Japan), while MPC was obtained from NOF Corporation (Tokyo, Japan). Sodium peroxodisulfate as a water soluble radical initiator and 2, 2'-azobis(2,4-dimethyl valeronitrile) (ADVN) as an oil soluble radical initiator were purchased from Wako Pure Chemicals. Polyvinyl alcohol (degree of polymerization $=$ 500 , saponification value $=86.5-89 \mathrm{~mol} \%$ ) was purchased from Nacalai Tesque (Kyoto, Japan), while dibutyl phthalate and sodium dodecyl sulfate were purchased from Wako Pure Chemicals. Solvents utilized for the surface modification, methanol $(\mathrm{MeOH})$, ethanol (EtOH), 2-propanol (IPN), acetonitrile (ACN), acetone (A), and tetrahydrofuran (THF) were purchased from Wako Pure Chemicals as of the highest grade.

\subsection{Apparatus}

Scanning electron micrographs were obtained using a Keyence scanning electron microscope (SEM), VE-8800 (Osaka, Japan) after Au-Pd sputtering by a Vacuum Device, MSP-1S sputtering device (Mito, Japan). Infrared spectra were recorded on a JASCO Corporation, FT/IR-4200 (Tokyo, Japan) using KBr method as well as ATR method. The surface and porosity of particles were measured by nitrogen adsorption porosimetry using a Gemini 2360 (Micromeritics Instruments, Norcross, GA, USA). The specific surface areas were calculated using BET (Brunauer-Emmett-Teller) and the specific pore volumes were calculated by BJH (Barrett-Joyner-Halenda) method.

\subsection{Preparation of uniformly sized GDMA base particle} Uniformly sized GDMA base particle was prepared by multi-step swelling and polymerization method according to the method reported previously [9]. First of all, 0.27 $\mathrm{mL}$ of uniformly sized polystyrene seed particles $(0.053$ $\mathrm{g} / \mathrm{mL}$ ) dispersed in water was mixed with micro-emulsion prepared from $0.025 \mathrm{~g}$ of sodium dodecyl sulfate, $10 \mathrm{~mL}$ of water and $0.41 \mathrm{~mL}$ of dibutyl phthalate as an activating solvent by sonication. This first swelling step was carried out at room temperature overnight with stirring at $125 \mathrm{rpm}$ until the micro-droplets of oil were completely disappeared.

Next, the dispersion of ADVN as the initiator, $5.0 \mathrm{~mL}$ of toluene as a porogen, $5.0 \mathrm{~mL}$ of water and $20 \mathrm{~mL}$ of $3.0 \%$ polyvinyl alcohol aqueous solution as a dispersion stabilizer was added to the dispersion of swollen particles. This second step swelling step was carried out at room temperature overnight with stirring at $125 \mathrm{rpm}$. Next, the dispersion of GDMA $(5.0 \mathrm{~mL})$ as the crosslinker, $15 \mathrm{~mL}$ of water, and $20 \mathrm{~mL}$ of $3.0 \%$ polyvinyl alcohol aqueous solution was added to the dispersion of swollen particles. This third swelling step was carried out at room temperature overnight with stirring at $125 \mathrm{rpm}$. After the third swelling step was completed, the polymerization procedure was started at $75{ }^{\circ} \mathrm{C}$ for $24 \mathrm{hr}$ under argon atmosphere with slow stirring. The resulting polymer particles were washed, collected and dried as reported previously [9].

\subsection{Surface modification of GDMA based particles}

In $25 \mathrm{~mL}$ of each organic solvent (methanol, ethanol, 2-propanol, acetonitrile, acetone, or THF) $0.75 \mathrm{~g}$ of MPC as the surface modifying monomer and $0.03 \mathrm{~g}$ of ADVN as an initiator were added to observe the solubility of MPC into each solvent. After the observation, MPC was polymerized at elevated temperature for $24 \mathrm{hr}$ to observe solubility of resulting poly-MPC into the solvent. After removal of the solvent, the amount of precipitated poly-MPC was measured.

In above mentioned MPC system, $2.5 \mathrm{~g}$ of GDMA based particles was added for the surface modification. After $24 \mathrm{hr}$ polymerization, the MPC-modified particles were washed, collected, and dried to measure the weight of the particles. The recovery was calculated by a standard method.

\subsection{Chromatographic evaluation}

The surface modified particles as well as base GDMA particles were packed into a stainless steel column $(4.6 \mathrm{~mm}$ I. D. X $150 \mathrm{~mm}$ ) by a slurry method using a Chemco packing system 124A (Osaka, Japan). The LC system was composed of an LC-20AT pump, an SPD-M20A spectrometer, a system controller, CBM-20A operated by an LC-solution (all from Shimadzu, Kyoto, Japan) and a Rheodyne 7125 valve loop injector (Rheodyne, Cotati, CA, USA). Column temperature was kept at $35^{\circ} \mathrm{C}$ by using a CTO-20AC column oven (Shimadzu) and detection was 


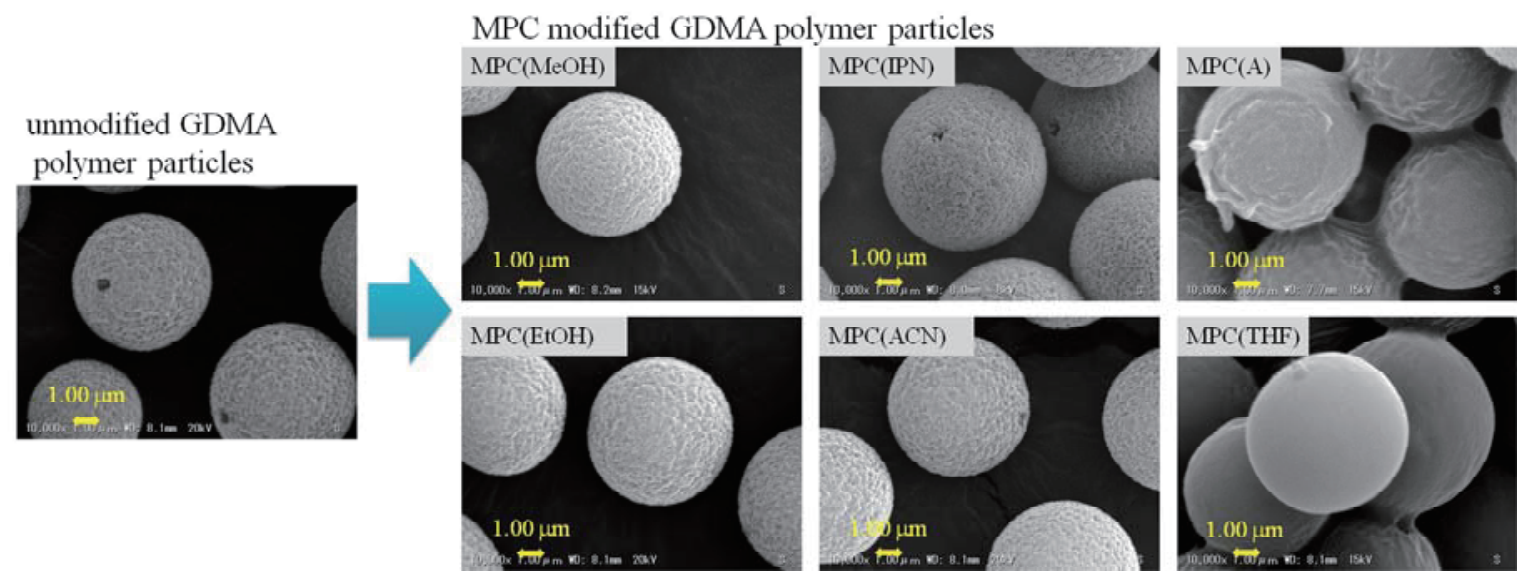

Fig. 2. SEM Pictures of MPC modified particles.

performed at $254 \mathrm{~nm}$. The retention factor $(k)$ at 0.8 $\mathrm{ml} / \mathrm{min}$ was calculated using the equation $k=\left(t_{R}-t_{0}\right) / t_{0}$, where $t_{R}$ and $t_{0}$ are the retention times of the retained and unretained solutes, respectively. There data were obtained in triplicate.

\section{Results and discussion}

\subsection{Solubility of $M P C$ and poly-MPC}

As the results of solubility tests, MPC (monomer) was soluble in $\mathrm{MeOH}$, EtOH, IPN, and ACN, while acetone and THF did not dissolve MPC completely. After the polymerization, as summarized in Table 1, poly-MPC (PMPC) was dissolved in methanol and ethanol, and up to $60 \%$ of the polymer was precipitated from an alcoholic solvent, 2-propanol. On the other hand, in acetonitrile, acetone, as well as THF, poly-MPC was precipitated almost quantitatively. According to these observations, in alcoholic solvents, poly-MPC dominantly remained in the solvent to result in less amount of the polymer could be introduced on the surface of GDMA based particles with shorter chain compared those in acetone and THF.

Table 1. Precipitation of Poly-MPC (PMPC).

\begin{tabular}{ccccccc}
\hline Solvent & MeOH & EtOH & IPN & ACN & A & THF \\
\hline $\begin{array}{c}\text { Precipitated } \\
\text { PMPC (\%) }\end{array}$ & 1.6 & 2.8 & 40.5 & 99.2 & 108.6 & 96.9 \\
$\begin{array}{c}\text { PMPC in } \\
\text { solvent (\%) }\end{array}$ & 98.4 & 97.2 & 59.5 & 0.8 & 0.0 & 3.1 \\
\hline
\end{tabular}

\subsection{Surface modification to GDMA based particles}

The surface modification onto GDMA base particles was performed in above mentioned 6 solvents. First of all, the recovery of particles is listed in Table 2 .

As expected based on the solubility of MPC and poly-MPC, around $70 \%$ of recoveries were obtained in the alcoholic solvents. Acetone and THF afforded higher recoveries around $80 \%$ than that in acetonitrile. In addition, the resulting particles were aggregated in acetone and THF. Again, poly-MPC is not dissolved in these three solvents.

Table 2. Recovery of PMPC modified GDMA polymer particle.

\begin{tabular}{ccccccc}
\hline Solvent & $\mathrm{MeOH}$ & $\mathrm{EtOH}$ & $\mathrm{IPN}$ & $\mathrm{ACN}$ & $\mathrm{A}$ & $\mathrm{THF}$ \\
\hline $\begin{array}{c}\text { Recovery } \\
(\%)\end{array}$ & 68.3 & 69.3 & 70.5 & 69.5 & 83.7 & 84.6 \\
\hline
\end{tabular}

According to SEM observation in Fig. 2, nicely prepared GDMA base particles having size uniformity clearly showed a trace of the seed polymer on the surface of each GDMA particle. After the polymerization in $\mathrm{MeOH}$ and $\mathrm{EtOH}$, surface morphology of the particles can hardly be changed because of high solubility of poly-MPC in these two solvents.

Similarly, in IPN and ACN, surface morphology was slightly changed and the trace of seed particle looked to be clogged up. On the other hand, acetone and THF resulted in completely different surface morphologies, smoothly covered surfaces with the traces of aggregations between particles.

A qualitative analysis of the modified particles by differential IR spectra was summarized in Fig. 3. A positive peak around $960 \mathrm{~cm}^{-1}$ indicated in ellipse attributed to an ammonium group [10] was observed on all spectra. These facts suggested that the surface modification by MPC was successfully done in all 6 solvents. Although almost no change of the surface morphology was found in those in methanol as well as in acetonitrile, relatively large peaks were observed on the particles modified in methanol and acetonitrile.

Table 3 summarized BET surface area and total pore volume of the prepared particles. Compared with unmodified GDMA, BET surface areas and total pore 

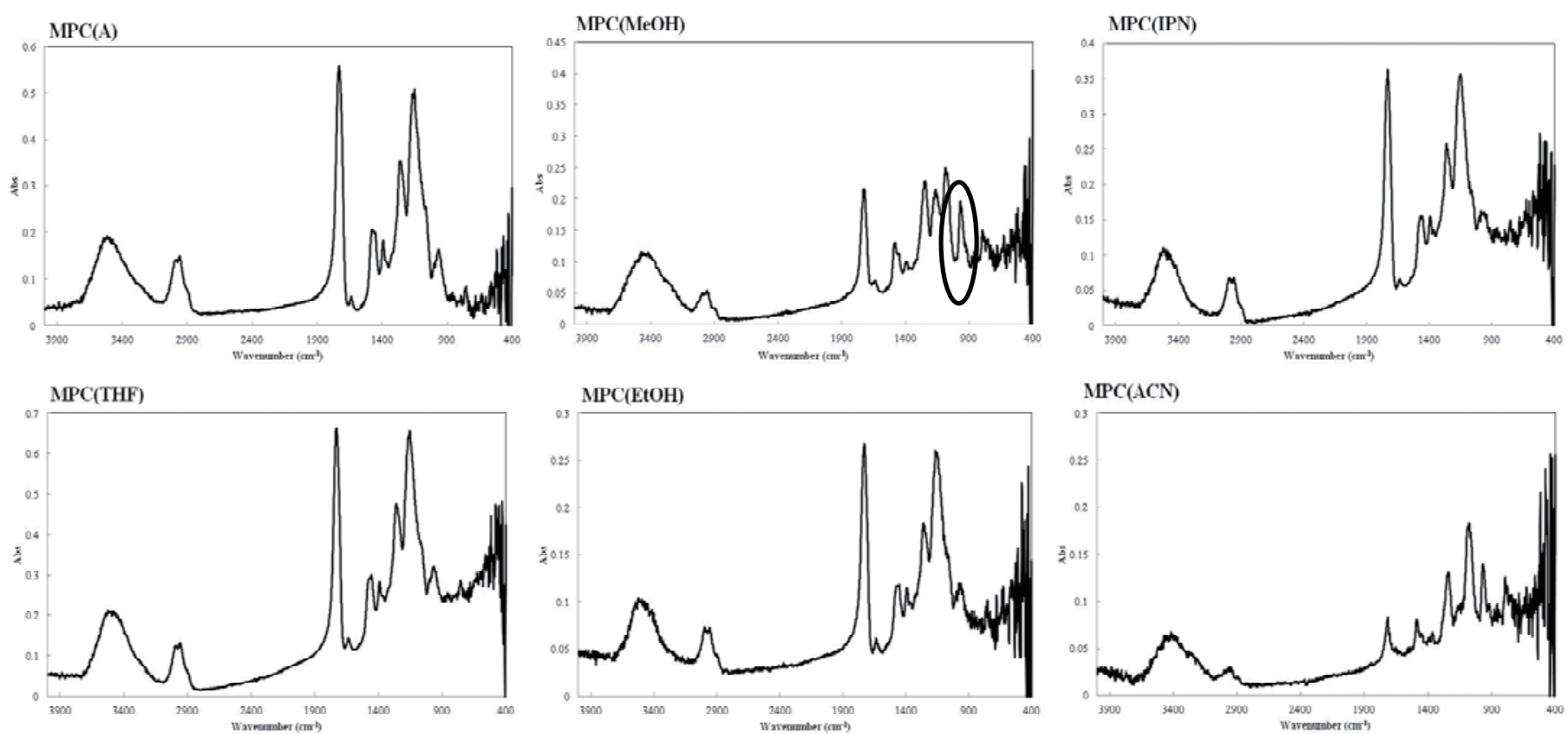

Fig. 3. Differential IR Spectra of the modified particles.

volumes of those modified in acetone, PMPC (A) and in THF, PMPC (THF) were greatly reduced to result in almost non-porous morphology. These facts were supported by greatly changed surface morphology as indicated in Fig. 2 and surface covered particles were obtained.

Table 3. BET surface area and total pore volume of prepared particles

\begin{tabular}{ccc}
\hline sample & $\begin{array}{c}\text { BET surface } \\
\text { area }\left(\mathrm{m}^{2} / \mathrm{g}\right)\end{array}$ & $\begin{array}{c}\text { total pore volume } \\
\left(\mathrm{cm}^{3} / \mathrm{g}\right)\end{array}$ \\
\hline unmodified GDMA & 111.57 & 0.054 \\
PMPC (MeOH) & 100.46 & 0.049 \\
PMPC (EtOH) & 97.73 & 0.048 \\
PMPC (IPN) & 68.24 & 0.033 \\
PMPC (MeCN) & 77.83 & 0.038 \\
PMPC (A) & 4.55 & 0.002 \\
PMPC (THF) & 8.89 & 0.005 \\
\hline
\end{tabular}

As suggested in Table 2, recoveries of the modified particles in methanol (PMPC (MeOH)), ethanol (PMPC $(\mathrm{EtOH}))$, 2-propanol (PMPC (IPN)), and acetonitrile (PMPC $(\mathrm{ACN})$ were close each other, around $30 \%$ of BET surface areas and total pore volumes of PMPC (IPN) and PMPC (ACN) were reduced, while tiny changes were found on PMPC (MeOH) and PMPC (EtOH). Only a speculative explanation satisfying the obtained facts in this study is illustrated in Fig. 4. According to the good solubility of poly-MPC in methanol and ethanol, very short chains of MPC are introduced in the pores, while relatively long chains of MPC modified the inner surface of GDMA base particles. [11]

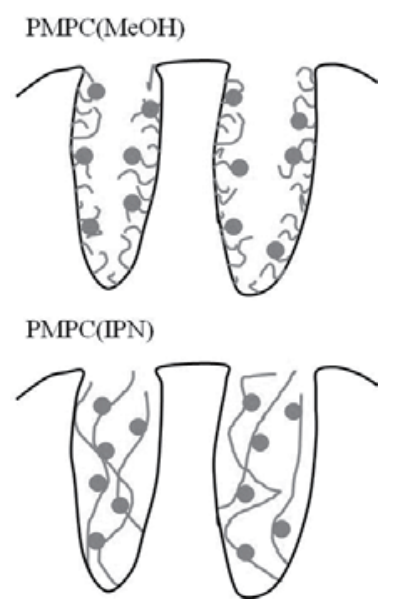

Fig. 4. Image of PMPC on the surface of base particle.

\subsection{Chromatographic evaluation}

Except PMPCs (A) and (THF) due to aggregation, other four PMPCs (MeOH), (EtOH), (IPN) and (ACN) including unmodified GDMA were chromatographically evaluated. First of all, a planar recognition ability was evaluated by separation factor $\alpha\left(k_{2} / k_{1}\right)$ for a planar solute, triphenylene and a sterically bulky solute, $o$-terphenyl as depicted in Fig. 5. By comparison with unmodified GDMA, MPC 
modified four particles showed higher $\alpha(\mathrm{T} / \mathrm{O})$ values. Especially, PMPC (IPN) and PMPC (ACN) resulted in significantly larger $\alpha(\mathrm{T} / \mathrm{O})$ values than those on PMPC $(\mathrm{MeOH})$ and PMPC (EtOH). These observations strongly support the speculative illustration (Fig. 4) [9,12-14].

A separation factor $\alpha$ ( $\left.k_{\text {caffeine }} / k_{\text {phenol }}\right)$ reveals a hydrophilicity of the stationary phase in reversed-phase HPLC. Fig. 6 clearly showed PMPC (IPN) and PMPC (ACN) had much lower hydrophilic interaction compared with that on unmodified GDMA. On the contrary, that on PMPC (MeOH) afforded larger value by comparison with that on even unmodified GDMA. Both of minus and plus charges on MPC is balanced out through intermolecular interaction between MPCs, therefore, poly-MPC chains on PMPC (IPN) and PMPC (ACN) were close each other, while those on PMPC (MeOH) and PMPC (EtOH) were rather isolated each other. These estimations will also satisfy the illustration in Fig 4.

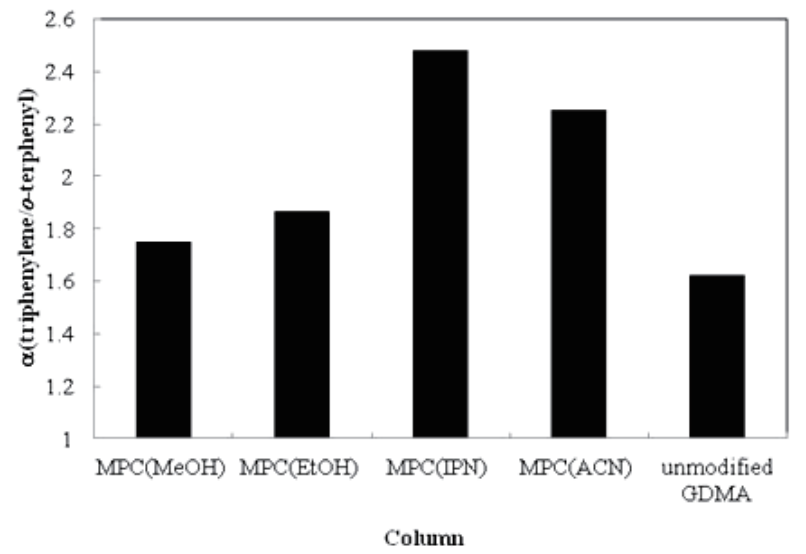

Fig. 5. Planar recognition ability of the modified particles (Mobile phase; $10 \mathrm{mmol}$ phosphate buffer $(\mathrm{pH}=2.6)$ / acetonitrile $=40 / 60)$.

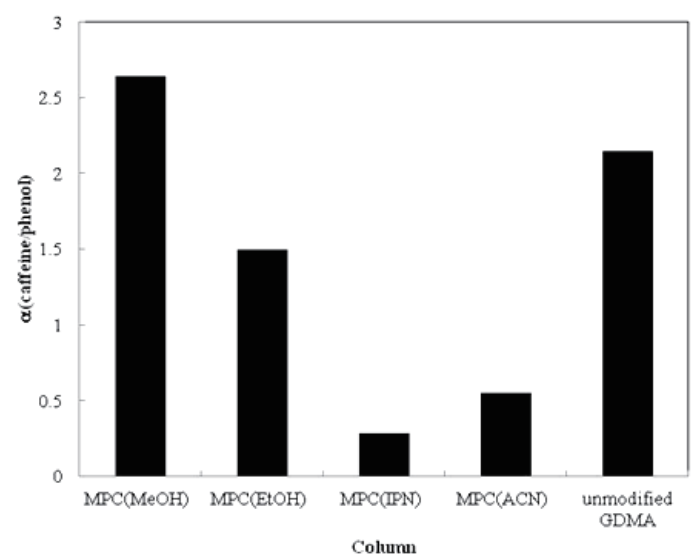

Fig. 6. Hydrophilic properties of the modified particles (Mobile phase; acetonitrile).
As mentioned in introduction section, as a pre-concentration phase for extraction of hydrophilic environmental pollutants, washing process using organic mobile phase such as acetonitrile is essential for removal of hydrophobic environmental contaminants. Therefore, retention properties for an acidic solutes as well as a basic solute were evaluated in absolute acetonitrile mobile phase with phenol as a phenolic solute as well as hydrophobic benzene and toluene. The results were depicted in Fig. 7.

Interestingly, MPC (IPN) and MPC (ACN) showed larger $k$ values for an acidic solute, benzoic acid. On these particles, all the observations suggested closely interacted MPC side chains, that means an ammonium group located on the top of MPC interacted with benzoic acid. On the contrary, MPC (MeOH) showed larger $k$ value for a basic solute, pyridine. With all the particles, hydrophobic solutes, benzene and toluene afforded almost no retention. These observations strongly support the speculative illustration (Fig. 4)

\section{Conclusion}

To control effects of the ion-exchange groups in HPLC, the solvents utilized for surface modification of MPC were changed. The solubility of MPC as well as poly-MPC into the selected solvent unexpectedly affected surface property of the modified polymer particle to result in almost opposite chromatographic characteristic of poly-MPC. The present paper will provide a rather simple method useful for surface modification by functional monomer(s).

\section{Acknowledgments}

This study was support in part by JSPS KAKENHI Grant Number 15H03846 and BYQ funding from 2013 to 2015.

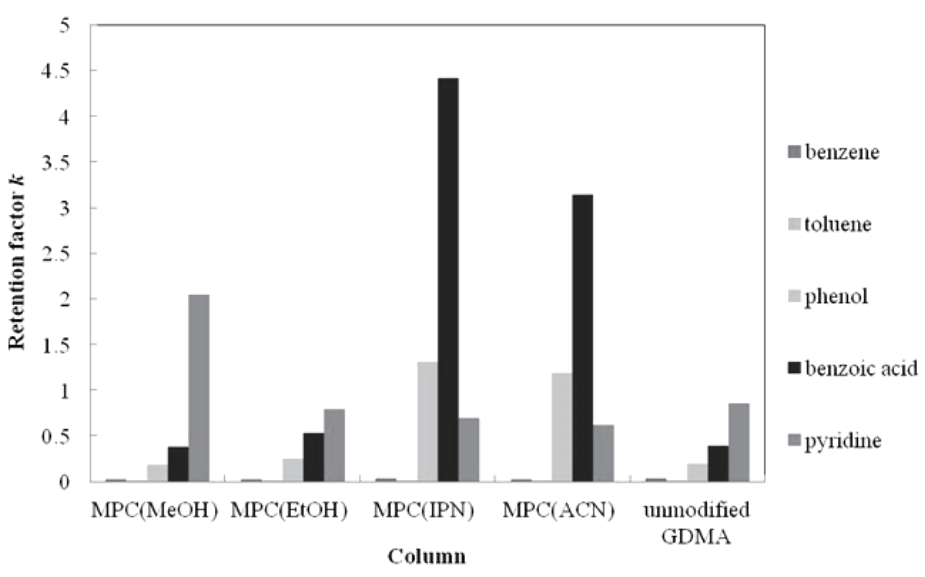

Fig. 7. Chromatographic properties of the modified particles (Mobile phase; acetonitrile). 


\section{References}

[1] Kubota,R.; Tahara, M.; Shimizu, K.; Nishimura, T. Bull. Natl. Inst. Health Sci. 2008, 126, 98-103.

[2] Nelson, E.D.; Do, H.; Lewis, R. S.; Carr, S.A. Environ. Sci. Technol. 2011, 45, 1228-1234.

[3] Hauswirth, S. C.; Birak, P. S.; Rylander, S. C.; Miller, C. T. Environ. Sci. Technol. 2012, 46, 426-433.

[4] Kimata, K.; Iwaguchi, K.; Onishi, S.; Jinno, K.: Eksteen, R.; Hosoya, K.; Araki, M.; Tanaka, N. J. Chromatogr. Sci. 1989, 27, 721-728.

[5] Hosoya, K.; Mori, T.; Sakamoto, M.; Kubo, T.; Kaya, K. Chromatographia 2009, 70, 699-704.

[6] Hosoya, K.; Hira, N.; Yamamoto, K.; Nishimura, M.: Tanaka, N. Anal. Chem. 2006, 78, 5729-5735.

[7] Tanaka, N.; Kimata, K.; Mikawa, Y.; Hosoya, K.; Araki, T. J. Chromatogr. 1990, 535, 13-31.

[8] Hosoya, K. Chromatography 2013, 34, 1-22.

[9] Hosoya, K.: Teramachi, T.; Tanaka, N.; Kobayashi, A.; Kanda, T.; Ohtsu, Y. Anal. Chem. 2001, 73, 5852-5857.

[10] Hesse, M.; Meier, H.; Zeeh, B.; Nomura, M.; Baba, A.; Miura, M.; Spectroscopic Methods in Organic Chemistry, Kagaku-Dojin Publishing Company, INC. 2008; Chapter 2.

[11] Hosoya, K.; Kubo, T.; Takahashi, K.; Ikegami, T.; Tanaka, N. J. Chromatogr. A 2002, 979, 3-10.

[12] Tanaka, N.; Hashizume, K.; Araki, M. J. Chromatogr. 1987, 400, 33-45.

[13] Mori, T.; Kubo, T.; Hosoya, K. Anal. Sci. 2010, 26, 311-316.

[14] Tanigawa, T.; Kubo, T.; Hosoya, K. Chem. Lett. 2012, 41, 1265-1266.

[15] Tanigawa, T. Doctoral thesis, Tohoku University, 2013. 